\title{
CHIP-mediated CIB1 ubiquitination regulated epithelial- mesenchymal transition and tumor metastasis in lung adenocarcinoma
}

\author{
Yuanqi Liu $\mathbb{1}^{1,2} \cdot$ Yanwu Zhou ${ }^{1,2} \cdot$ Pengfei Zhang ${ }^{3} \cdot$ Xizhe Li $^{1,2} \cdot$ Chaojun Duan ${ }^{1,2} \cdot$ Chunfang Zhang ${ }^{1,2,4}$
}

Received: 19 September 2019 / Revised: 30 September 2020 / Accepted: 5 October 2020 / Published online: 20 October 2020

(c) The Author(s) 2020. This article is published with open access

\begin{abstract}
CIB1 is a homolog of calmodulin that regulates cell adhesion, migration, and differentiation. It has been considered as an oncogene in many tumor cells; however, its role in lung adenocarcinoma (LAC) has not been studied. In this study, the expression levels of CIB1 in LAC tissues and adjacent normal tissues were examined by immunohistochemistry, and the relationship between CIB1 expression and patient clinicopathological characteristics was analyzed. The effects of CIB1 on epithelial-mesenchymal transition (EMT), migration, and metastasis of LAC cells were determined in vitro and vivo. Proteins interacting with CIB1 were identified using electrospray mass spectrometry (LS-MS), and CHIP was selected in the following assays. Carboxyl-terminus of Hsp70-interacting protein (CHIP) is a ubiquitin E3 ligase. We show that CHIP can degrade CIB1 via promoting polyubiquitination of CIB1 and its subsequent proteasomal degradation. Besides, lysine residue 10 and 65 of CIB1 is the ubiquitinated site of CIB1. Furthermore, CHIP-mediated CIB1 downregulation is critical for the suppression of metastasis and migration of LAC. These results indicated that CHIP-mediated CIB1 ubiquitination could regulate epithelial-mesenchymal and tumor metastasis in LAC.
\end{abstract}

\section{Introduction}

These authors contributed equally: Yuanqi Liu, Yanwu Zhou

These authors jointly supervised this work: Chaojun Duan, Chunfang Zhang

Edited by D. Aberdam.

Supplementary information The online version of this article (https:// doi.org/10.1038/s41418-020-00635-5) contains supplementary material, which is available to authorized users.

Chaojun Duan duancjxy@126.com

$\triangle$ Chunfang Zhang zcf6169@outlook.com

1 Department of Thoracic Surgery, Xiangya Hospital, Central South University, Changsha 410008, PR China

2 Hunan Engineering Research Center for Pulmonary Nodules Precise Diagnosis \& Treatment, Changsha 410008 Hunan, PR China

3 Department of Oncology, Xiangya Hospital, Central South University, Changsha 410008, PR China

4 National Clinical Research Center for Geriatric Disorders, Changsha 410008 Hunan, PR China
Lung cancer is one of the most common malignant tumors and poses a severe threat to human health [1]. LAC is the most common pathological type in the past few decades, and its incidence is still proliferating [2]. LAC with poor prognosis (with about 15\% 5-year survival and 30\% 5-year recurrence [3]) because the mechanism of LAC is not fully understood. Therefore, it is imperative to explore the molecular mechanisms of LAC and find new therapeutic targets.

Calcium and integrin binding 1 (CIB1) is a homolog of calmodulin initially found in yeast cells [4]. CIB1 is widely expressed in systemic tissues and acts as a ligand for the platelet integrin $\alpha \mathrm{IIb} \beta 3$ tail cell [5]. CIB1 can promote cell proliferation, migration, and inhibition of apoptosis in various cancer by regulating several kinase partners or oncogenic signaling $[6,7]$. In the early stage of this project, the LAC and its matched adjacent normal tissues were analyzed by liquid chromatography-tandem mass spectrometry and found that CIB1 was highly expressed in the LAC [8]. However, the mechanism underlying CIB1 in LAC is unknown. Since ubiquitination plays an important role in the regulation of protein expression, we sought to find the regulation of CIB1 through ubiquitinate pathway. 
The degradation of the target protein by the ubiquitinproteasome pathway requires three enzymes: ubiquitinactivating enzyme 1 (E1), ubiquitin-binding enzyme 2 (E2), and ubiquitin ligase E3 (E3). Ubiquitin is activated by E1 and transported to E2. E2 interacts directly or indirectly with a specific E3, which recognizes a specific substrate and transports the activated ubiquitin to one or more lysine residues of the substrate. The polyubiquitin chain allows the protein to be recognized by the $26 \mathrm{~S}$ proteasome, where the protein is deubiquitinated and unfolded and eventually degraded [9]. E3 plays a crucial role in the degradation of the entire protein, determining the specificity of the reaction [10]. In this study, we found CIB1 is a novel substrate of carbonyl-terminus of hsp70-interacting protein (CHIP) which is an E3 ubiquitin ligase and closely linked with many oncogene-encoded proteins [11]. Moreover, the interaction between CIB1 and CHIP is closely linked to LAC.

\section{Methods and materials}

\section{Ethics approval and consent to participate}

This study was approved by the ethics committee of Xiangya Hospital, Central South University, and written informed consent was obtained from all patients (Grant number: 201703317). The Animal Ethics Committee approved all animal studies (Grant number: 2018sydw0221) and undertaken following the official recommendations of the Care and Use of Laboratory Animals of Xiangya Hospital, CSU.

\section{Patients and tissue specimens}

A total of 60 pairs of LAC tumor tissues were obtained from patients underwent lobectomy at the Xiangya Hospital, CSU between July 2010 and December 2011. All patients were followed up every 3 months by telephone or a visit by our research team for survival and recurrence inquiry until death or until the end of the investigation.

\section{Cell culture and reagents}

PC-9, A549, H1299, H1975, H1437, HEK293, and BEAS2B were obtained from the Chinese Academy of Science Cell Bank (Shanghai, China). Five LAC cell lines were cultured in RPMI-1640 (12633012, Gibco, Billings, MT, USA). HEK293 were cultured in DMEM (11965084, Gibco). BEAS-2B were cultured in BEGM Kit (CC-3170, Lonza/Clonetics Corporation). All the above mediums were supplemented with $10 \%$ FBS (10437-028, Gibco), $100 \mathrm{U} / \mathrm{mL}$ penicillin, and $100 \mu \mathrm{g} / \mathrm{mL}$ streptomycin (15070063, Gibco). All cell lines were passaged less than ten times after the initial revival from frozen stocks. All cell lines were authenticated prior to use by short tandem repeat profiling by Genesky Biotechnologies (Shanghai, China). AKT inhibitor MK-2206 was obtained from MCE (MK-2206, MedChemExpress, Monmouth Junction, NJ, USA).

\section{Plasmid and transfection}

Flag-CIB1, Myc-CHIP, Myc-CHIP- $\Delta$ Ubox, HA-ubiquitin plasmid were purchased from GeneChem (Shanghai, China) by cloning the corresponding human full-length DNA sequence into the $3 \times$ Flag or MYC or HA-pcDNA 3.1(+), and its mutant expression plasmid was generated by in-fusion cloning kits (TSV-S2, Tsingke, Beijing, China). Three shRNAs were constructed, and CIB1-RNAi (35732-1) was selected according to the knockdown efficiency (Supplementary Fig. S1A, Supplementary Table S1). Also, an efficient CHIPRNAi has been selected among three constructed shRNA (Supplementary Fig. S1B, Supplementary Table S1). All mutation primers were listed in Supplementary Table S2. All transfection experiments using Lipofectamine 3000 reagents (L3000015, Invitrogen, Eugene, OR, USA) under the manufacturer's protocols.

\section{Viruses and transduction}

CIB1, CHIP, CHIP- $\Delta$ Ubox expression, and sh-CHIP lentivirus were purchased from Vigene (Vigene, Shandong, China). The transfection methods were performed according to the manufacturer's protocols. The transfected cells were screened by $2 \mu \mathrm{g} / \mathrm{ml}$ puromycin (ST551, Beyotime, Beijing, China) to obtain a stably expressed cell line.

\section{Immunoprecipitation and MS}

The cell lysate for immunoprecipitation was prepared in two different methods. The cell lysate used for ubiquitin modification analysis was prepared by the denatured method. The cells plate $(150 \times 25 \mathrm{~mm})$ were first washed by PBS and added by $0.4 \mathrm{ml}$ denatured cell lysis buffer which contains 50 $\mathrm{mM}$ Tris $(\mathrm{PH}=7.5)$ and $70 \mathrm{mM} \beta$-Mercaptoethanol (pre-boil for $10 \mathrm{~min}$ before use). Then the cells were scraped and transferred to a $2.5 \mathrm{ml}$ tube, followed by $10 \mathrm{~min}$ boiled. The cells lysis was later added by four volumes $(1.6 \mathrm{ml})$ of $1 \mathrm{X}$ pre-chilled cell lysis buffer (9803S, Cell signaling, Danvers, MA, USA) and be prepared for immunoprecipitation. Nondenatured cell lysis was prepared using Western \&IP lysis buffer (P0013, Beyotime) containing 1X Protease inhibitor cocktail without EDTA (HY-K0010, MedChemExpress).

For co-immunoprecipitation, the supernatant was incubated with corresponding antibody overnight $(2 \mu \mathrm{g}$ antibody per $500 \mu \mathrm{g}$ protein sample) and with protein $\mathrm{A}+\mathrm{G}$ magnetic beads (HY-K0202, MedChemExpress) for $3 \mathrm{~h}$ at $4{ }^{\circ} \mathrm{C}$. HA-specific magnetic beads (HY-K0201, MedChemExpress) were used 
for HA-fusion protein. Antibodies used in immunoprecipitation are described below: Ubiquitin (sc-8017, Santa Cruz, Dallas, TX), CIB1(11823-1-AP, Protein Tech, Chicago, IL), Flag (66008-3-Ig, Protein Tech). The magnetic beads were isolated by the magnetic racket and washed by phosphate buffered saline supplemented with $0.5 \%$ Triton-100 (PBST). Samples were eluted with $0.1 \mathrm{M}$ glycine $\mathrm{pH} 3.0$ adjusted to $\mathrm{pH}$ 7.5 with Tris buffer and run on an SDS-PAGE. After SDSPAGE electrophoresis, proteins in the gels were detected by silver staining and followed by in-gel trypsin digestion and MS analysis as previously described by us [12, 13].

\section{In vitro ubiquitylation assay}

$2.5 \mu \mathrm{l}$ 10× E3 Ligase Reaction Buffer (B-71, Boston Biochem), $1 \mu \mathrm{l}$ Recombinant Human Ubiquitin Protein (U-100H-10M, Boston Biochem), $10 \mathrm{mM}$ MgATP Solution (B-20, Boston Biochem), $100 \mathrm{nM}$ Recombinant Human His6-Ubiquitin E1 Enzyme (E-304-05, Boston Biochem), $1 \mu \mathrm{M}$ Recombinant Human UbcH5a/UBE2D1 Protein (E2-616-100, Boston Biochem), $1 \mu \mathrm{M}$ Recombinant Human CHIP/STUB1 Protein (E3-220-050, Boston Biochem), $5 \mu \mathrm{M}$ CIB1 Fusion Protein (Ag2391, Protein Tech) was added in a microcentrifuge tube and be incubated in a $37^{\circ} \mathrm{C}$ water bath for $60 \mathrm{~min}$. Then the lysis was added by SDS-PAGE sample buffer and followed by electrophoresis. The SDS-PAGE were then transferred onto PVDF membranes or be stained by sliver staining kits.

\section{Western blot}

Cells were lysed using Western \&IP lysis buffer (P0013, Beyotime) supplemented with 1X Protease inhibitor cocktail without EDTA (HY-K0010, MedChemExpress). Approximately $25 \mu \mathrm{g}$ of protein extracts were separated by $10 \%$ SDSPAGE, transferred onto PVDF membranes (ISEQ00010, Millipore). Then the PVDF membrane was blocked with $5 \%$ non-fat milk and incubated with specific antibodies. The antibodies used in WB are described below: CIB1 (1:1000, ab220606, Abcam), CHIP (1:1000, ab134064, Abcam), anti-E-cadherin (1:1000, ab76055, Abcam), anti-Ncadherin (1:1000, ab18203, Abcam), anti-Vimentin (1:1000, ab92547, Abcam), anti-Ubiquitin (1:1000, 3933S, Cell signaling), anti-Tublin (1:1000, 2144, Cell signaling), anti-Flag (20543-1-AP, Protein Tech), anti-HA (66006-2-Ig, Protein Tech) and anti-Myc (60003-2-Ig, Protein Tech). An enhanced chemiluminescent (Millipore) chromogenic substrate was used to visualize the bands.

\section{Quantitative real-time PCR}

TRIzol was used in the extraction of Total RNA. cDNA was generated using primeScript ${ }^{\mathrm{TM}}$ RT reagent Kit with gDNA Eraser (RR047B, Takara, Shangdong, China). The RNA levels were quantified using SYBR Green qPCR Mix (RR820B, Takara). Primers for CIB1, CHIP, and $\beta$-actin were list in Supplementary Table S2. The expression level of according target relative to that of $\beta$-actin was defined as $-\Delta \mathrm{CT}=-(\mathrm{CT} \mathrm{CIB} 1 / \mathrm{CHIP}-\mathrm{CT} \beta$-actin $)$.

\section{Histology immunohistochemistry and immunofluorescence}

Tumor sections were processed following standard methods and examined using routine H\&E staining. For immunohistochemistry, antigen retrieval was done using standard citrate buffer protocol. The expression was graded as follows: negative (score 0), weak (score 1), moderate (score 2), and strong (score 3). Percentage scores were assigned as 1 , $1-25 \%$; 2, 26-50\%; 3, 51-75\%; and 4, 76-100\%.

For immunofluorescence, cells were the culture in 24Well plant and overnight. Also, antigen retrieval was done using standard protocol. The IHC and IF using the following antibodies: CIB1(1:200, ab56664, Abcam), CHIP (1:200, ab134064, Abcam), anti-E-cadherin (1:200, ab76055, Abcam), anti-N-cadherin (1:200, ab18203, Abcam), anti-Vimentin (1:200, ab92547, Abcam).

\section{Experimental metastasis in vivo}

A549 ${ }^{\text {luc }}$ with lentivirus $\left(1 \times 10^{6}\right.$ in $100 \mu \mathrm{l}$ PBS $)$ infection were injected into 5-week-old nude male mice via the tail vein. Three nude mice were included in each group. Intraperitoneal injection of D-Luciferin potassium salt was used in locating and monitoring the neoplasm (PerkinElmer, Waltham, MA, USA). The mice were sacrificed at 42 days after the injection.

\section{Dimensional scratch assays and transwell assays}

The $10 \mu \mathrm{l}$ pipette tip was used to draw a straight line in the sixwell plate with cells wholly fused. The initial and unhealed width was measured after replacing the serum-free medium. Three replicate wells were made in each group of cells, and each of the wells was randomly selected from three different visual fields for three times, and the average value was taken.

Cell $\left(4 \times 10^{4}\right)$ were seeded into upper chambers purchased from BD bioscience (BD Biosciences, NJ, USA) using FBS-free medium. The lower chamber was filled with medium supplemented with $20 \%$ FBS. After co-cultured in $37^{\circ} \mathrm{C}$ for $24 \mathrm{~h}$, the lower surface of the upper chambers was fixated and counted.

\section{Statistical analysis}

Data statistics in this study were statistically analyzed using SPSS 23. Comparisons between groups were analyzed by the $t$-test and $\chi^{2}$ test. The correlation between the expression 
level of CIB1 and the clinical features of LAC patients was analyzed by Spearman rank correlation analysis. The overall survival and disease-free survival curves of patients were drawn by the Kaplan-Meier method. In this study, $P<0.05$ was set as a critical value for comparing whether there was a significant difference between the groups.

\section{Result}

\section{CIB1 is frequently upregulated in LAC tissue and cell lines and associated with poor clinicopathological features and prognosis}

In our previous study, a total of 36 differentially expressed membrane proteins were found. We then compare those identified proteins using PubMed and ONCOMINE database. CIB1 was selected as our major research object due to a higher averaged ratio-fold change $(5.191 \pm 0.056)$. Analyzes of available ONCOMING data sets from normal versus LAC tissues showed that CIB1 levels are significantly upregulated in LAC patients (Fig. 1A). The Immunohistochemical assay showed that CIB1 protein was highly expressed in LAC compared to normal tissues, mainly in the cytoplasm and cell membrane (Fig. 1B). According to the scoring criteria mentioned in the previous method, CIB1 protein was highly expressed in 60\% (36/60) of LAC tissues and 30\% in normal tissues, the difference was statistically significant (Supplementary Table S3). The qRT-PCR showed that the overall mRNA expression of CIB1 in LAC tissues was significantly higher than that in normal lung tissues (Fig. 1C), of which 29 (48.3\%) in LAC tissues were highly expressed (higher than
A

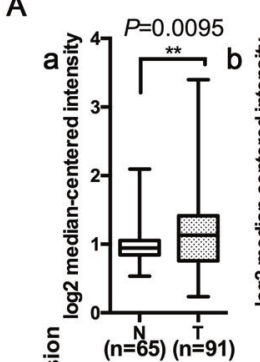

D
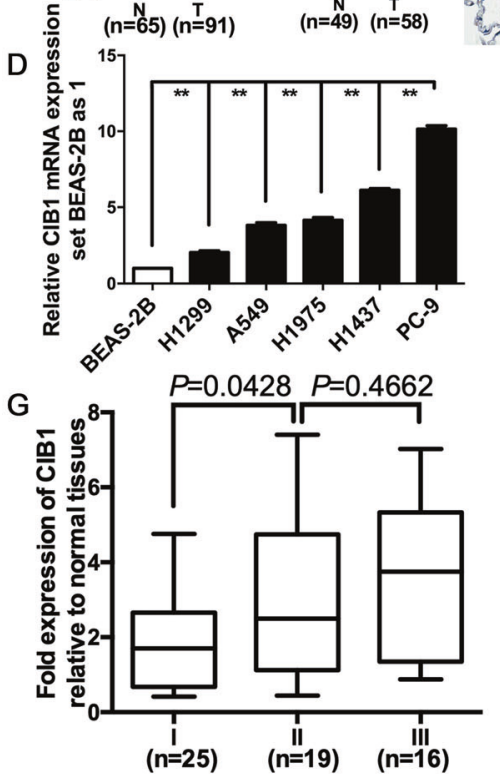

B

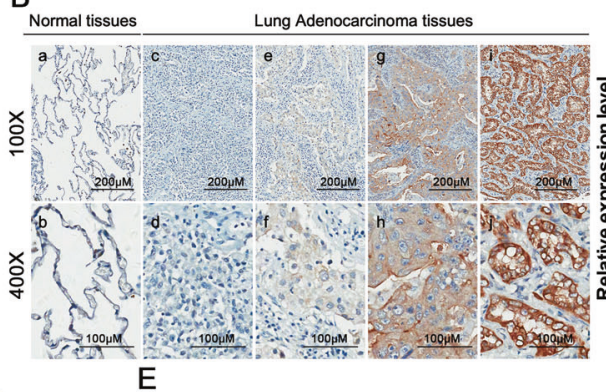

E

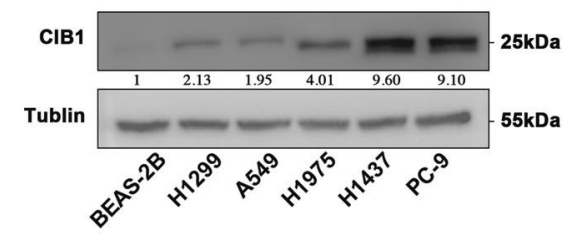

$\mathrm{H}$

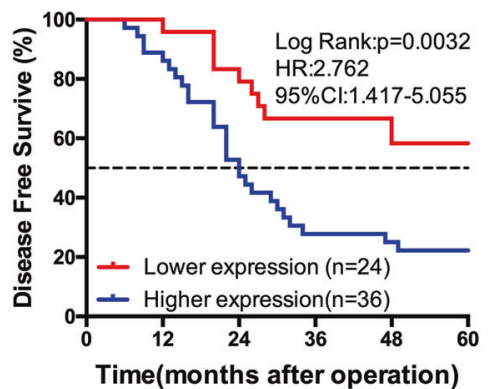

C
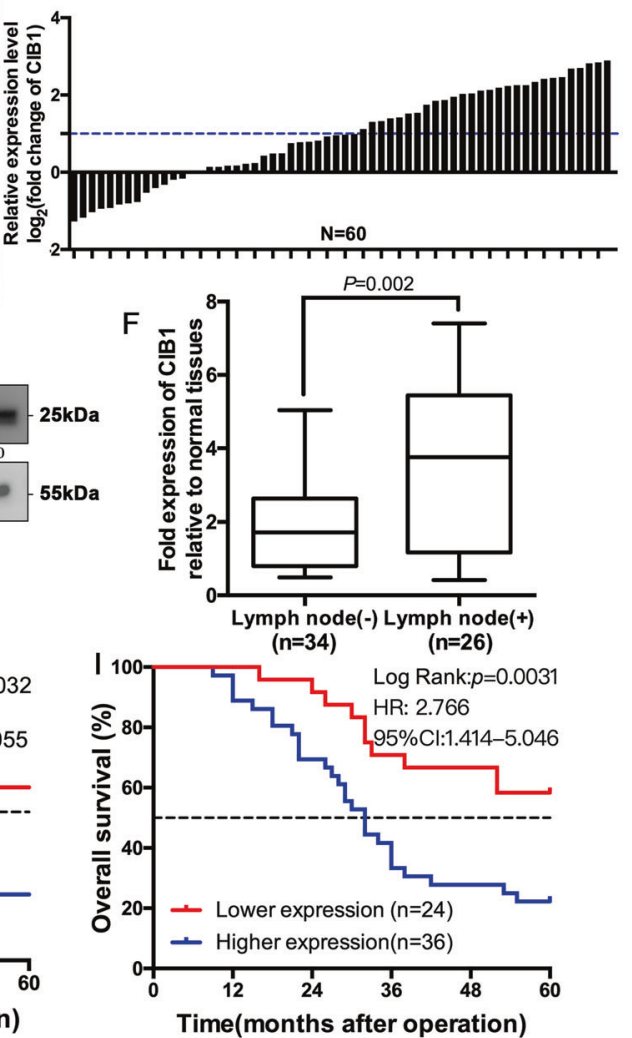

Fig. 1 CIB1 is frequently upregulated in LAC tissue and cell lines and associated with poor clinicopathological features and prognosis. A Representative statistics analysis of CIB1 levels in two different data using the ONCOMINE database (www.oncomine.org). Data were filtered to display upregulation of CIB1 in LAC tissues compared to normal tissues (T: Tumor, N: normal tissues). a: Hou Lung Statistics; b: Landi Lung Statistics. B Representative immunohistochemically stained images of normal and LAC tissue using anti-CIB1 antibody: a, b: negative expression of CIB1 in normal lung tissue; $\mathrm{c}, \mathrm{d}$ : negative expression of CIB1 in lung adenocarcinoma; e, f: weakly positive expression of CIB1 in lung adenocarcinoma; $\mathrm{g}, \mathrm{h}$ : moderately positive expression of CIB1 in lung adenocarcinoma; $\mathrm{i}, \mathrm{j}$ : Strong positive expression of CIB1 in lung adenocarcinoma;. C Relative mRNA expression level of CIB1 in LAC tumor tissue compare to normal tissues from 60 patients. D Quantitative RT-PCR results showed CIB1 expression levels in five LAC cell lines and normal bronchus cell lines. E Western blots results showed CIB1 expression levels in five LAC cell lines and normal bronchus cell lines. F, G Metastatic or advanced TNM stage LAC tumors had higher CIB1 expression levels compared with those of nonmetastatic or early TNM stage LAC tumors. H, I Kaplan-Meier analysis showed that the 5-year overall survival and disease-free survival for LAC patients with higher CIB1 expression levels were significantly shorter than those of patients with lower CIB1 expression level. $* P<0.05$; $* * P<0.01$; $* * * P<0.001$; HR, hazard ratio. 
two times, $\log 2$ (fold change) $>1$ ). Also, an elevated CIB1 expression was found in PC-9, H1437, H1299, H1975, and A549 cell lines compared to BEAS-2B by qRT-PCR and WB $P<0.01$, Fig. 1D, E).

We divided the patients into two groups according to the expression level of CIB1 in tumor tissue. Clinical data of two groups are collected and compared. High expression of CIB1 was associated with $\mathrm{N}$ stage $(P=0.019)$, TNM stage $(P=$ 0.045) (Table 1, Supplementary Table S5). What's more, CIB1 expression was significantly higher in LAC with lymph node metastasis and advanced stage (Fig. 1F, G). Moreover, patients with high CIB1 expression in tumor tissues had shorter overall survival and disease-free survival than patients with low CIB1 expression $(P<0.001$, Fig. 1H, I, Supplementary Fig. S2). In the COX proportional hazard regression model, the overall survival was associated with TNM stage $(P=0.042), \mathrm{CIB} 1$ expression $(P=0.058)$ and disease-free survival were associated with TNM stage $(P=0.016)$, CIB1 expression $(P=0.036)$ (Table 2$)$. All those were suggesting that CIB1 is highly expressed in LAC and closely associated with poor clinical characteristic and prognosis.

\section{CIB1 promotes LAC migratory capacity, metastatic ability, and induce EMT via AKT pathway in vitro}

Clinical characteristic demonstrated that overexpression of CIB1 is positive related to tumor metastatic. Therefore, we

Table 1 Correlation analysis between CIB1 expression and clinical characteristics.

\begin{tabular}{lllll}
\hline Variables & $n$ & \multicolumn{2}{l}{ CIB1 expression } & $P$ \\
\cline { 5 - 5 } & & Low & High & \\
\hline Age (years) & & & & 0.598 \\
$\quad \leq 55$ & 30 & 11 & 19 & \\
$\quad>55$ & 30 & 13 & 17 & \\
Gender & & & & 1.00 \\
$\quad$ Female & 25 & 12 & 18 & \\
$\quad$ Male & 35 & 12 & 18 & \\
Smoking history (years) & & & & 0.752 \\
$\quad \leq 10$ & 31 & 13 & 18 & \\
$>10$ & 29 & 11 & 18 & \\
Tumor differentiation & & & & 0.261 \\
$\quad$ High & 19 & 8 & 11 & \\
Medium & 29 & 9 & 20 & \\
Low & 12 & 7 & 5 & \\
T stage & & & & \\
$\quad$ T1 & 30 & 13 & 17 & \\
T2 & 18 & 7 & 11 & \\
T3 & 12 & 4 & 8 & \\
N stage & & & & 0.031 \\
$\quad$ N0 & 34 & 18 & 16 & \\
$\quad$ N1-2 & 26 & 6 & 20 & \\
TNM stage & & & & 0.045 \\
$\quad$ I & 28 & 15 & 13 & \\
II-III & 32 & 9 & 23 & \\
\hline
\end{tabular}

access the ability of CIB1 in the metastatic capacity in LAC cells. Four cell lines, A549/H1299 (with lower CIB1 expression) and PC-9/H1437 (with higher CIB1 expression), were picked to perform the further study.

The transwell assays and wound healing assays to evaluate the ability of LAC metastasis and migration. Overexpression of CIB1 accelerates A549 and H1299 cell migration in wound healing and transwell assay (Fig. 2A, B, E, Supplementary Fig. S3). Meanwhile, knocking down the expression of CIB1 in PC-9 and H1437 cells, the ability of according migration was also weakened in the following transwell assays and wound healing assays (Fig. 2C, D, E; Supplementary Fig. S3).

Studies have proved that overexpression of CIB1 can lead to abnormal activation of AKT, thereby promoting the development of tumor cells [6]. Since AKT pathway plays a central role in a variety of oncogenic processes including cell growth, proliferation, apoptotic cell death, motility, angiogenesis, and metastasis [14], we further test activation of AKT in transfected LAC cell. The WB result showed that in LAC cells, CIB1 could activate the AKT pathway by promoting phosphorylation of AKT in LAC (Fig. 2F). Also, activation of $\mathrm{PI} 3 \mathrm{~K} / \mathrm{AKT}$ is a central feature of EMT development [15]. Further WB showed that the EMT marker expression was altered after CIB1 downregulation in PC-9 cells and H1437 cells, including downregulation of $\mathrm{N}$-cadherin and Vimentin and upregulation of E-cadherin (Fig. 2F). When CIB1 was upregulated in H1299, and A549 cells by transfection, $\mathrm{N}$-cadherin, and Vimentin expression increased, and E-cadherin expression decreased (Fig. 2F). Therefore, we hypothesize that CIB1 may promote EMT marker expression in LAC cells via PI3K/AKT pathway, contributing to the increased migration and invasion abilities that ultimately lead to the tumorigenesis. To further test this hypothesis, we subjected correspondent transfected LAC cells to the sublethal dose of MK2206, a tested AKT inhibitor. We found the compromised upregulated expression of N-cadherin and Vimentin and excessive E-cadherin protein production in CIB1 transfected $\mathrm{H} 1299$ and A549 cell lines after treated with MK-2206 (5 $\mu$ M, 24 h). Further, the adverse effects were also observed in the MK2206 treated CIB1 downregulated cell lines (Fig. 2F). The results indicate that CIB1 can promote EMT via the AKT pathway in LAC cells.

\section{CHIP interacts with $\mathrm{CIB} 1$ and reduces the protein level of CIB1}

To identify CIB1-interacting proteins, immunoprecipitation assay using anti-CIB1 or IgG antibody followed by electrospray mass spectrometry (LS-MS) was used. The proteins met the following criteria were considered as differentially expressed proteins between the two types of 
Table 2 Cox regression analysis of overall survival (OS) and disease-free survival (DFS) in 60 patients with LAC cancer.

\begin{tabular}{|c|c|c|c|c|c|c|c|c|}
\hline & \multicolumn{4}{|l|}{ Univariate analysis } & \multicolumn{4}{|l|}{ Multivariate analysis } \\
\hline & \multicolumn{2}{|l|}{ DFS } & \multicolumn{2}{|l|}{ OS } & \multicolumn{2}{|l|}{ DFS } & \multicolumn{2}{|l|}{ OS } \\
\hline & HR $(95 \%$ CI $)$ & $P$ & $\mathrm{HR}(95 \% \mathrm{CI})$ & $P$ & HR $(95 \% \mathrm{CI})$ & $P$ & HR $(95 \% \mathrm{CI})$ & $P$ \\
\hline Age & $1.135(0.600-2.146)$ & 0.698 & $1.023(0.537-1.952)$ & 0.944 & $1.275(0.615-2.642)$ & 0.359 & $1.089(0.523-2.268)$ & 0.819 \\
\hline Adjuvant therapy & $0.643(0.340-1.218)$ & 0.175 & $0.604(0.316-1.154)$ & 0.127 & $0.435(0.173-1.091)$ & 0.076 & $0.523(0.207-1.324)$ & 0.171 \\
\hline Gender & $1.315(0.694-2.493)$ & 0.401 & $1.199(0.628-2.289)$ & 0.582 & $1.980(0.938-4.178)$ & 0.073 & $1.720(0.824-3.592)$ & 0.149 \\
\hline \multicolumn{9}{|l|}{ Tumor differentiation } \\
\hline High & 1 & & 1 & & 1 & & 1 & \\
\hline Middle-low & $1.116(0.558-2.234)$ & 0.756 & $1.039(0.516-2.091)$ & 0.916 & $0.680(0.283-1.634)$ & 0.388 & $0.688(0.277-1.706)$ & 0.419 \\
\hline Low & $0.405(0.132-1.243)$ & 0.114 & $0.402(0.131-1.236)$ & 0.112 & $0.460(0.126-1.676)$ & 0.239 & $0.513(0.143-1.843)$ & 0.306 \\
\hline \multicolumn{9}{|l|}{ Tumor size } \\
\hline $\mathrm{T} 1$ & 1 & & 1 & & 1 & & & \\
\hline $\mathrm{T} 2$ & $1.426(0.674-3.020)$ & 0.354 & $1.424(0.673-3.011)$ & 0.356 & $1.250(0.491-3.182)$ & 0.639 & $1.289(0.500-3.325)$ & 0.600 \\
\hline T3 & $2.426(1.082-5.439)$ & 0.031 & $1.927(0.845-4.395)$ & 0.119 & $1.848(0.712-4.797)$ & 0.207 & $1.440(0.551-3.763)$ & 0.456 \\
\hline Lymphatic metastasis & $5.952(2.968-11.936)$ & 0.000 & $6.950(3.384-14.273)$ & 0.000 & $1.714(0.508-5.781)$ & 0.385 & $2.081(0.578-7.483)$ & 0.262 \\
\hline \multicolumn{9}{|l|}{ TNM classification } \\
\hline I & 1 & & & & 1 & & 1 & \\
\hline II & $2.555(1.098-5.944)$ & 0.029 & $2.288(0.968-5.411)$ & 0.059 & $1.720(0.634-4.668)$ & 0.287 & $1.447(0.502-4.175)$ & 0.494 \\
\hline III & $10.216(4.167-25.051)$ & 0.000 & $10.551(4.171-26.688)$ & 0.000 & $6.040(1.391-26.228)$ & 0.016 & $4.738(1.054-21.296)$ & 0.042 \\
\hline CIB1 expression & $2.834(1.370-5.864)$ & 0.005 & $2.696(1.299-5.596)$ & 0.008 & $2.531(1.064-6.019)$ & 0.036 & $2.316(0.973-5.513)$ & 0.058 \\
\hline
\end{tabular}

immunoprecipitated: proteins were identified based on $\geq 2$ peptides with $\geq 95 \%$ confidence and showed an averaged ratio-fold change $\geq 1.5$ (the student's $t$ test, $P<0.05$ ) in the two LC-MS analyses. According to these criteria, a total of 38 differentially expressed proteins were found (Fig. 3A, Supplementary Table S4). Since ubiquitin-proteasome systems closely link to the degradation of protein, we choose CHIP, the only E3 ubiquitin ligase being identified, for the following assays.

In PC-9 cell lines, immunoprecipitated using anti-CIB1 antibody followed by WB using anti-CHIP antibody showed the combination between CIB1 and CHIP (Fig. 3B). Immunofluorescence using CIB1 and CHIP antibodies in PC-9 and A549 cell lines showed that two proteins were co-expressed in the cell both in the cytoplasm and nucleus (Fig. 3H).

After observing the interaction between CIB1 and CHIP, we then found the suppressed endogenous and exogenously CIB1 was also observed in the CHIP overexpressing PC-9 and HEK293 cell lines (Fig. 3C, D). Moreover, in the HEK293 cell, we observed that CHIP could decrease CIB1 in a dose-dependent manner (Fig. 3G). Further immunohistochemical staining of 60 patients showed that CHIP is lowly expressed in tissues and is significantly negatively correlated with CIB1 expression (Fig. 3E, F). RT-qPCR results show that the mRNA expression levels of CIB1 in CHIP overexpressing PC-9 did not show any difference, indicating that CHIP regulates the stability of CIB1 protein in the post-transcriptional levels (Supplementary Fig. S4). We used CHX to measure the half-life of CIB1, showing that CHIP attenuates CIB1 stability (Fig. 3I). Overexpression of Myc-CHIP can downregulate the protein level of CIB1, but after adding MG-132 (proteasome inhibitor), the protein level of CIB1 downregulated by CHIP is reversed. However, and the protein level of CIB1 down regulated by CHIP was not reversed after treating with leupeptin (Fig. 3J). All those were indicating that CHIP-induced decline in CIB1 protein levels is dependent on the proteasome pathway.

\section{CHIP facilitates K48-linked polyubiquitination of CIB1}

After demonstrating that CHIP may promote CIB1 degradation through ubiquitination-proteasome pathways. We overexpressed CHIP and blank plasmids in PC-9 cells, followed by using CIB1 antibody to precipitate CIB1 protein and detected it with ubiquitin antibody. WB results showed that the level of ubiquitinated CIB1 is significantly higher in cells transfected with CHIP than in cells transfected with blank plasmid alone (Fig. 4A). Moreover, when flag-CIB1, HA-ubiquitin, and Myc-CHIP expression plasmid were co-transfected into HEK293T cells, the level of ubiquitination of flag-CIB1 was significantly increased (Fig. 4B). The U-box domain constitutes a loop finger structure (RNG-finger), which is a domain that functions as a ubiquitin ligase. To compare the ubiquitination of CIB1 by CHIP, we constructed the CHIP- $\Delta$ Ubox plasmid by deleting the Ubox domain of CHIP. We found that CHIP$\Delta$ Ubox mutants could not increase ubiquitination levels of CIB1, both endogenously and exogenously (Fig. 4C, D). Moreover, in vitro ubiquitination assays with recombinant CHIP and CIB1 showed that CHIP markedly enhances CIB1 ubiquitination (Fig. 4F), suggesting that CHIP 
A Negative control CIB1-upregulation

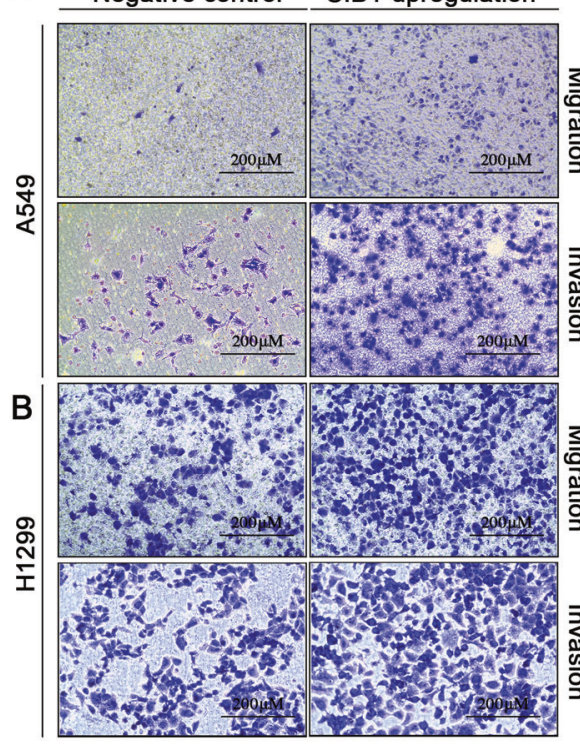

E
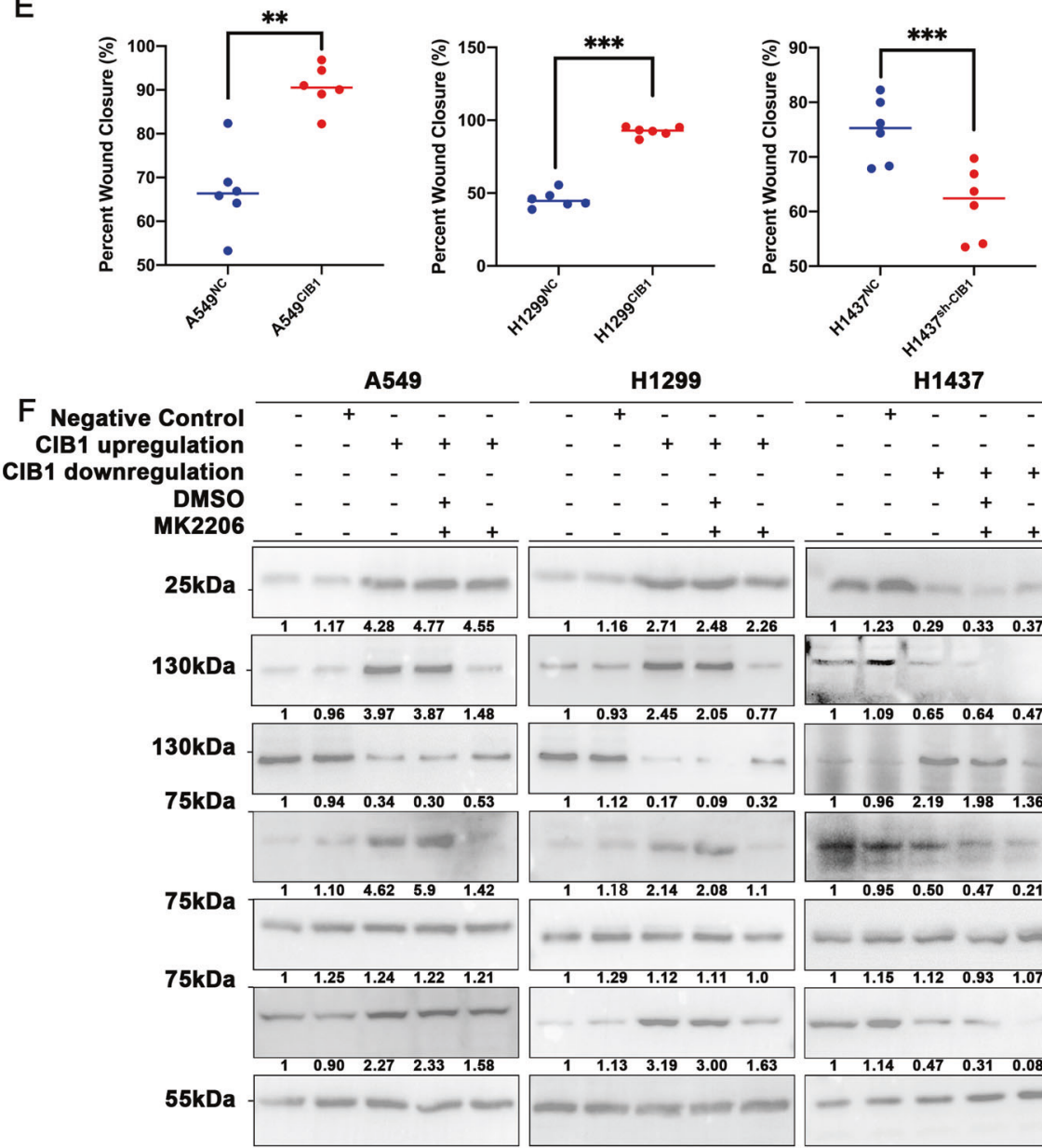

H1299

Fig. 2 CIB1 promote LAC migratory capacity, metastatic ability, and induce EMT via AKT pathway in vitro. A, B Representative images and quantification of the Transwell assay using CIB1upregulation A549/H1299 cells. C, D Representative images and quantification of the Transwell using anti-CIB1-transfected H1437/ PC-9 cells. E Quantification of wound healing assays using CIB1/anti-

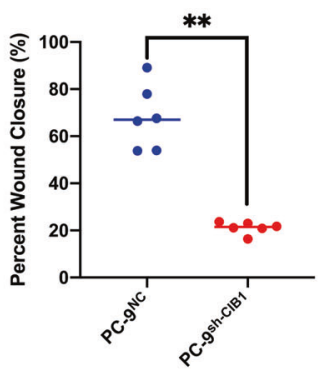

H1437

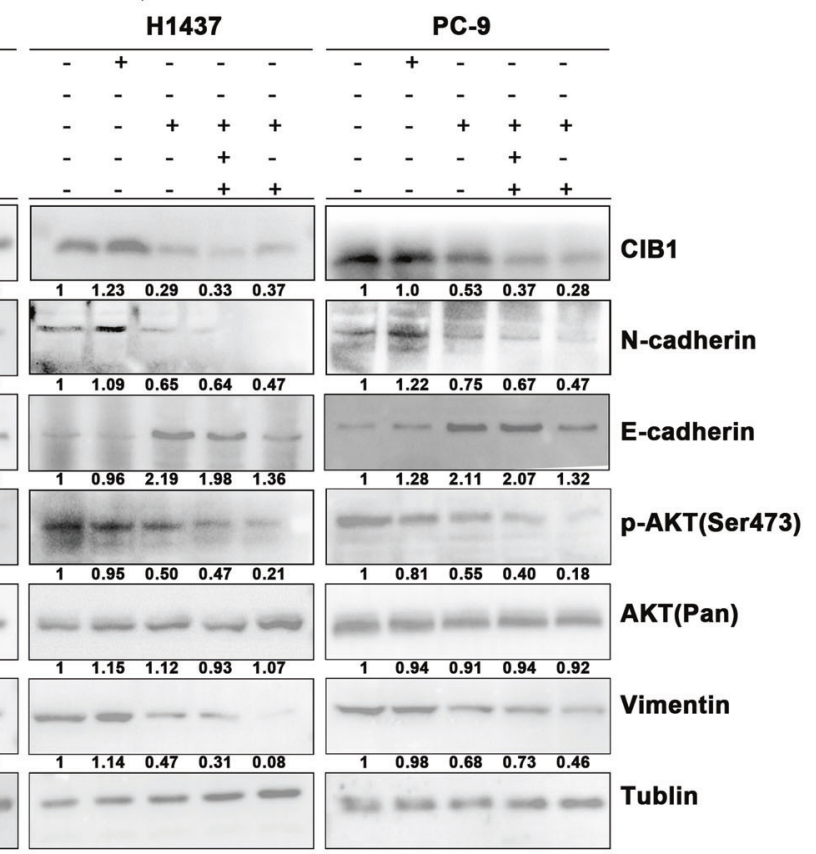

C ClB1-inhibition Negative control

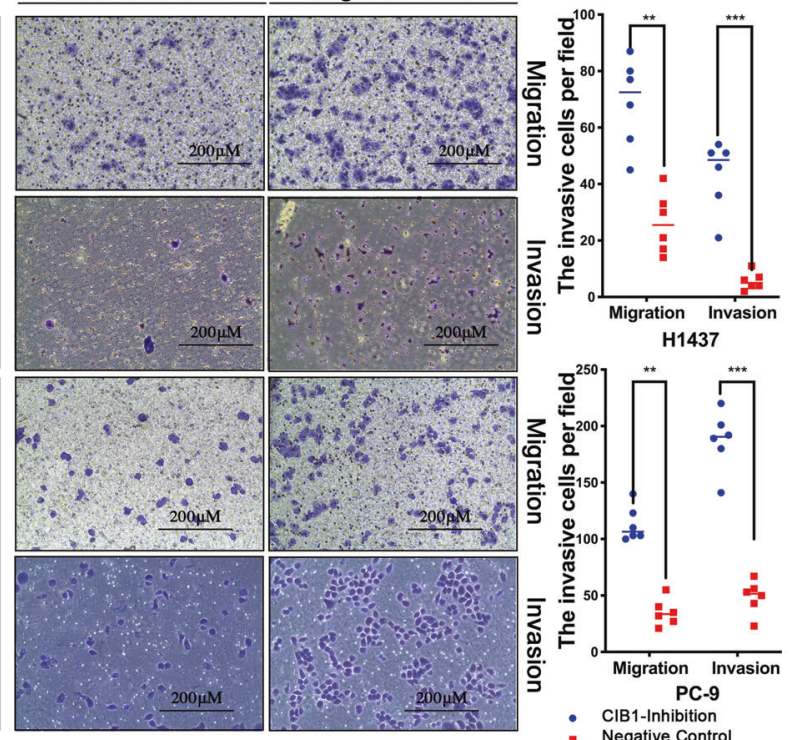

- CIB1-Inhibition

CIB1

CIB1-transfected LAC cells. F Western blot assay showing AKT (pan), p-AKT, E- cadherin, N-cadherin, and Vimentin protein level in CIB1/anti-CIB1-transfected LAC cells. Cells were treated with $5 \mu \mathrm{M}$ MK2206 for $24 \mathrm{~h}$. $* P<0.05$ vs negative control (NC) group, $* * P<$ 0.01 vs NC group, $* * * P<0.001$ vs $\mathrm{NC}$ group. 


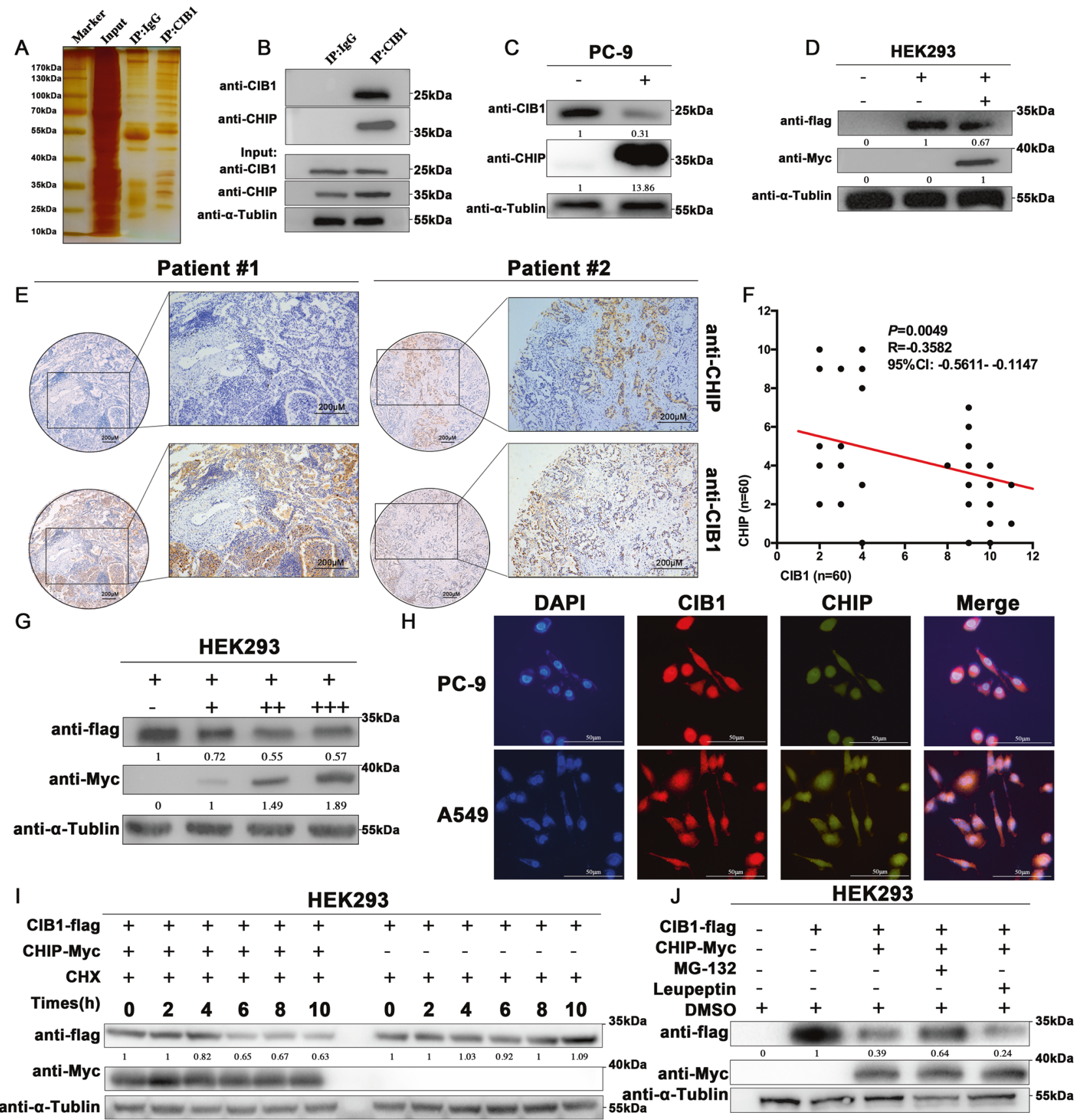

Fig. 3 CHIP interacts with CIB1 and decreases the protein level of CIB1. CIB1 and CHIP specifically interact. A SDS-PAGE gel of proteins bound to IgG (left lane) or CIB1 (right lane). Protein marker stands stand for (from top to bottom): 170, 130, 100, 70 (Red), 55, 40, $35,25,15 / 10 \mathrm{kDa}$. B Endogenous interaction between CIB1 and CHIP in PC-9 cells. C PC-9 cells were transiently transfected for $36 \mathrm{~h}$ with CHIP plasmid, and cell lysates were immunoblotted with indicated antibodies. D HEK293 cells were transiently transfected for $36 \mathrm{~h}$ with Flag-CIB1 and Myc-CHIP, and cell lysates were immunoblotted with indicated antibodies. E Representative immunohistochemically stained images of LAC tissues using the anti-CHIP and anti-CIB1 antibodies. Areas in the black squares are magnified in the right slide panels. F Pearson analysis correlation between CIB1 and CHIP expression in
LAC tissues $(r=-0.3582, P=0.0049)$ G HEK293 cells were transfected with Flag-CIB1 $(1 \mu \mathrm{g})$ and various concentrations of Myc-CHIP plasmids $(0,0.2,0.5$, and $1 \mu \mathrm{g})$. And after $36 \mathrm{~h}$, cell lysates were immunoblotted with anti-Flag, anti-Myc, and anti-Tublin antibodies. H Representative confocal images of immunostaining for CHIP (green) and CIB1 (red) in PC-9 and A549 cells. Scale bar, $50 \mu \mathrm{m}$. I Cells were treated with $40 \mu \mathrm{g} / \mathrm{ml}$ cycloheximide for the indicated times, and cell lysates were immunoblotted with indicated antibody. J HEK293 cells were transfected for $36 \mathrm{~h}$ with Flag-CIB1 alone or together with Myc-CHIP and treated with MG-132 $(10 \mu \mathrm{M})$, or leupeptin $(100 \mu \mathrm{M})$ for $4 \mathrm{~h}$. Cell lysates were analyzed by western blotting with the indicated antibodies. 


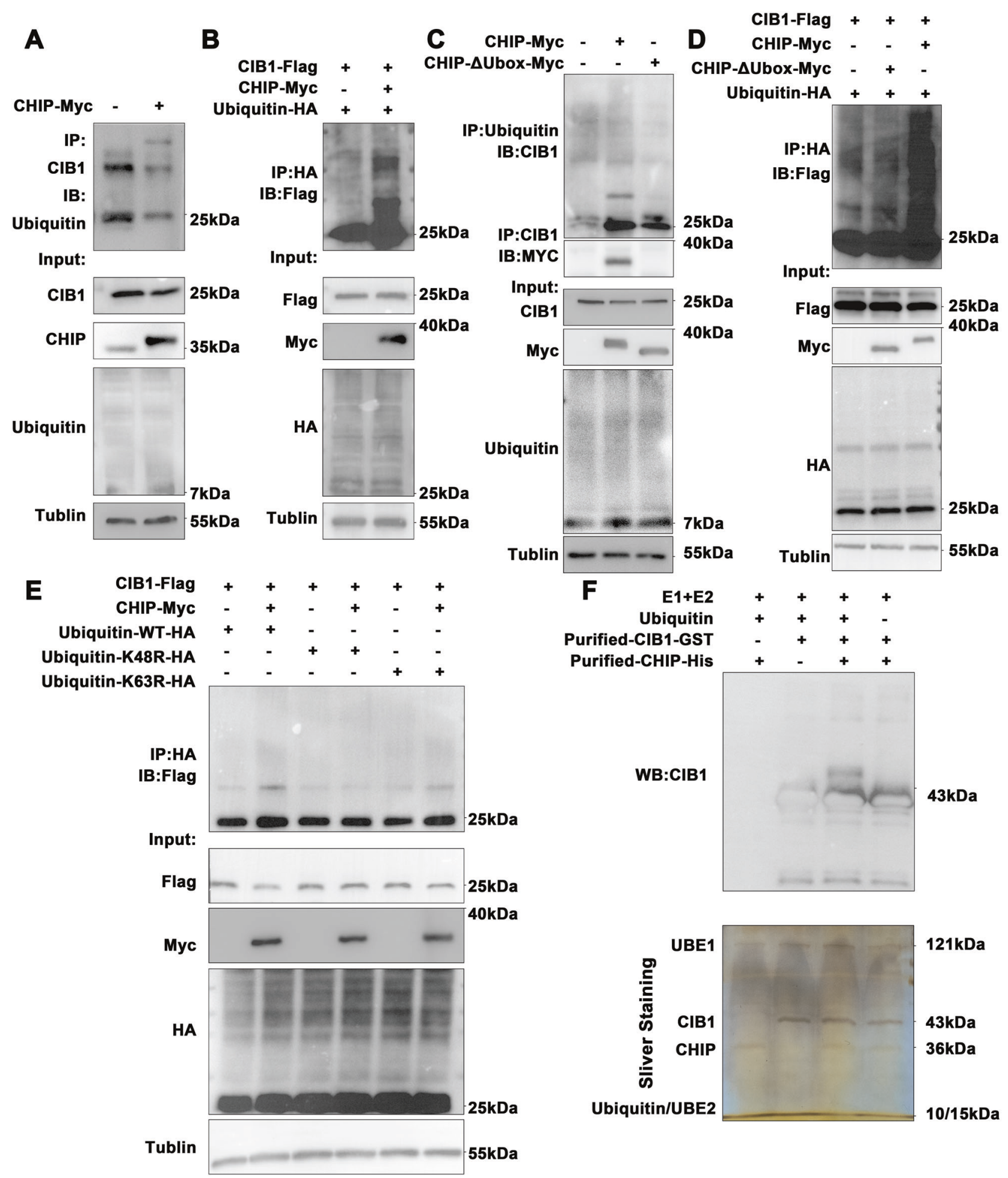

directly ubiquitinates CIB1. Subsequently, we transferred K48 and K63 into HEK293 cells, respectively. The CHIPmediated ubiquitination of CIB1 disappeared after the 48th lysine mutation on ubiquitin, while the K63 mutation did not change (Fig. 4E). All those were suggesting that CHIP promotes CIB1 ubiquitination via the Lys-48 residue of ubiquitin.

\section{CHIP targets CIB1 lysine 10 and lysine 65 for ubiquitination}

Since ubiquitination modification usually occurs in the lysine of a protein substrate, we further sought to explore which lysine on CIB1 may be the sites that cause ubiquitination modification. Eight lysine residues on the CIB1, 
Fig. 4 CHIP facilitates K48-linked polyubiquitination of CIB1. A PC-9 were transfected with CHIP plasmid for $36 \mathrm{~h}$. Cell extracts were immunoprecipitated with the anti-CIB1 antibody, followed by immunoblotting with the anti-Ubiquitin antibody. B HEK293 cells were transfected with HA-ubiquitin, Flag-CIB1 with or without MycCHIP. Cell lysates were immunoprecipitated with the anti-HA antibody, followed by immunoblotting with anti-flag antibodies. C PC-9 cells were transfected with CHIP or CHIP- $\Delta$ Ubox plasmid. Cell extracts were immunoprecipitated with the anti-ubiquitin antibody, followed by immunoblotting with the anti-CIB1 antibody. D HEK293 cell were transfected with Flag-CIB1, HA-ubiquitin, with or without Myc-CHIP or Myc -CHIP- $\Delta$ Ubox. Denatured cell lysates were immunoprecipitated with the anti-HA antibody, followed by immunoblotting with Flag antibodies. E Where specified, purified E1, E2, ubiquitin, CHIP and CIB1 proteins were incubated with in vitro ubiquitination buffers. Reaction samples were analyzed by SDSpolyacrylamide gel electrophoresis, followed by Sliver staining or immunoblotting with the indicated antibodies. F HEK293 cells were transfected for $36 \mathrm{~h}$ with Flag-CIB1, Myc-CHIP, HA-Ubiquitin or HAK48R-ubiquitin or HA-K63R-ubiquitin. Cell extracts were immunoprecipitated with the anti-HA antibody followed by immunoblotting with the anti-Flag antibody.

K10, K24, K65, K70, K83, K107, K150, K188 were mutated to ornithine (Supplementary Fig. S5), and cotransfected into HEK293 cells combine with HA-ubiquitin. Results showed that the ubiquitinated CIB1 was significantly decreased after lysine 10, and lysine 65 was mutated (Fig. 5A). Therefore, we speculate that lysine 10 and lysine 65 may be ubiquitination sites in CIB1 protein. It was further found that after the simultaneous mutation of $\mathrm{K} 10$ and K65, the ubiquitination of CIB1 showed a significant decrease compared with the individual mutations (Fig. 5B). Also, the Flag-CIB1 downregulation caused by CHIP overexpression was retrieved when K10 and K65 were co-mutated to ornithine (Fig. 5C). All those were suggesting that lysine 10 and lysine 65 may be the ubiquitinated site of CIB1 (Fig. 5D).

\section{CIB1 is negatively regulated by CHIP and affects the metastatic ability of lung adenocarcinoma cells in vitro}

After identifying the interaction between CIB1 and CHIP, we further examined the effectiveness of CHIP on the CIB1 mediated enhanced LAC migration and invasion in vitro. In A549 and H1299 cell lines, we simultaneously overexpressed both CIB1 and CHIP proteins using the corresponding plasmid. The transwell assays and wound healing assays were used to evaluate the invasion and migration ability. The results showed that the CIB1 induced LAC invasion, and metastasis of LAC was subdued by overexpressed CHIP, but CHIP- $\Delta$ Ubox did not produce the same effects (Fig. 6A-C, Supplementary Fig. S6). In additional, knocking down CHIP expression by its shRNA significantly enhanced LACs' migratory ability under
CIB1-overexpression conditions, indicating an enhanced cell migration was promoted by CHIP downregulation (Fig. 6A-C, Supplementary Fig. S6). Moreover, WB shows that p-AKT showed a significant turnover when CIB1 and CHIP were co-transfections in H1299 and A549 cell lines (Fig. 6D). Further, we exam the EMT marker (Vimentin, ECadherin, N-Cadherin) in those two transfected cells lines. The WB results show that the elevated N-Cadherin and Vimentin induced by CIB1 and reversed downregulated by CHIP overexpression, whereas the CHIP- $\Delta$ Ubox cannot have the same results. Moreover, the inhibition of E-cadherin caused by CIB1 overexpression can be overturned by CHIP upregulation but not through CHIP$\Delta$ Ubox (Fig. 6E). However, transfected-mediated expression of CHIP shRNA significantly boosted the CIB1-induced upregulation of N-cadherin and Vimentin protein, reversed that of E-cadherin protein (Fig. 6E). All those indicated that CIB 1 is negatively regulated by CHIP and affects the metastatic ability of lung adenocarcinoma cells in vitro.

\section{CIB1 is negatively regulated by CHIP and affects the metastatic ability of lung adenocarcinoma cells in vivo}

After found that CHIP can alleviate CIB1 induced LAC enhanced migratory capacity and metastasis ability in vitro, we further examine whether the same result can be observed in vivo. $\mathrm{A} 549^{\mathrm{NC}}$ and $\mathrm{A} 549^{\mathrm{CIB} 1}$ cells were injected into the tail vein of nude mice. Also, A549 ${ }^{\mathrm{CIB} 1 / \mathrm{CHIP}}$ and A549 ${ }^{\mathrm{CIB} 1 / \Delta \mathrm{Ubox}}$ were injected according to previously described methods. All the mice underwent bioluminescence imaging and were sacrificed for the subsequent assays after 42 days. Compared to the NC, A549 ${ }^{\mathrm{CIB} 1}$ injected mice had enhanced luminescence intensity. Also, A549 ${ }^{\mathrm{CIB} 1 / \mathrm{CHIP}}$ have lower node compare to A549 ${ }^{\mathrm{CIB} 1 / \mathrm{CHIP}-\Delta \text { Ubox }}$ (Fig. 7A-C). The same result was also observed in $\mathrm{HE}$ stains result. Moreover, IHC using Ncadherin and E-cadherin show corresponding alternation (Fig. 7D). Yet, another group of nude mice was sacrificed to test whether downregulating CHIP expression could greatly enhance the CIB1-overexpressed LAC migratory capacity and metastasis ability. It was notable that at 42 days following the tail vein rejection, an elevated metastasis node was observed in mice lung (Supplementary Fig. S7). Thus, we proved that CIB1 can negatively be regulated by CHIP and affects the metastatic ability of lung adenocarcinoma cells in vivo.

\section{Discussion}

CIB1 was initially thought to be a ligand for the platelet integrin $\alpha \mathrm{IIb} \beta 3$ tail cell [4] and a small intracellular protein 

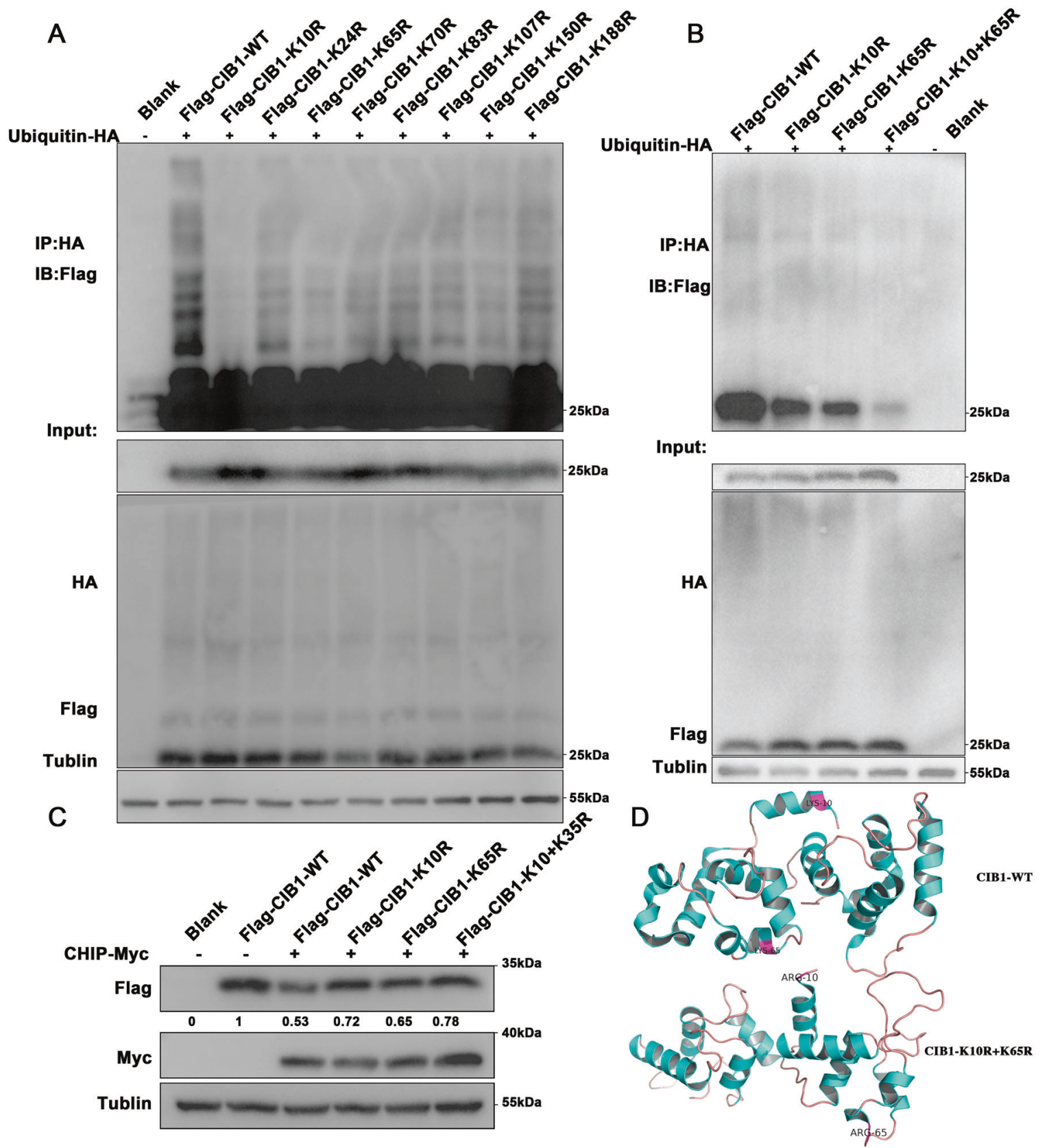

Fig. 5 Multiple $K$ residues of CIB1 are polyubiquitinated by CHIP. A HEK293 cells were co-transfected with HA-ubiquitin and Flag-CIB1 and its corresponding mutated plasmids for $36 \mathrm{~h}$. Cell lysates were subjected to immunoprecipitation with HA antibody, followed by Flag immunoblotting. B HEK293 cells were cotransfected with HA-ubiquitin with HA-ubiquitin, and Flag-CIB1 or Flag-K10R-CIB1 or Flag-K65R-CIB1 or Flag-K10R+K65R-CIB1.

that regulates kinase and integrin activity [5, 16-18]. CIB1 has been shown influenced many tumor cells activity via multiple signaling pathways $[7,19,20]$. In this study, based on previous reviews in our laboratory, we first explored the expression and clinical significance of CIB1 in LAC [8]. We found that CHIP can interact with CIB1 and degraded CIB1 through the ubiquitin-proteasome pathway. The
Cell lysates were subjected to immunoprecipitation with HA antibody, followed by Flag immunoblotting. C HEK293 cells were cotransfected with Flag-CIB1, Flag-K10R-CIB1, Flag-K65R-CIB1, Flag-K10R+K65R-CIB1 and Myc-CHIP. Cell lysates were analyzed by immunoblotting with MYC, Flag and $\alpha$-Tublin antibodies. D Computational modeling results show multiple binding sites for ubiquitin on CIB1. The red region is the binding site for CIB1.

interaction of CIB1 and CHIP then controlled the invasion and migration of lung adenocarcinoma.

We first showed that CIB1 is highly expressed in LAC and associated with adverse clinical feature and prognosis. Similar results were observed in hepatic carcinoma and triple-negative breast cancer $[6,7]$. After finding that the expression level of CIB1 is related to LAC metastasis, we 

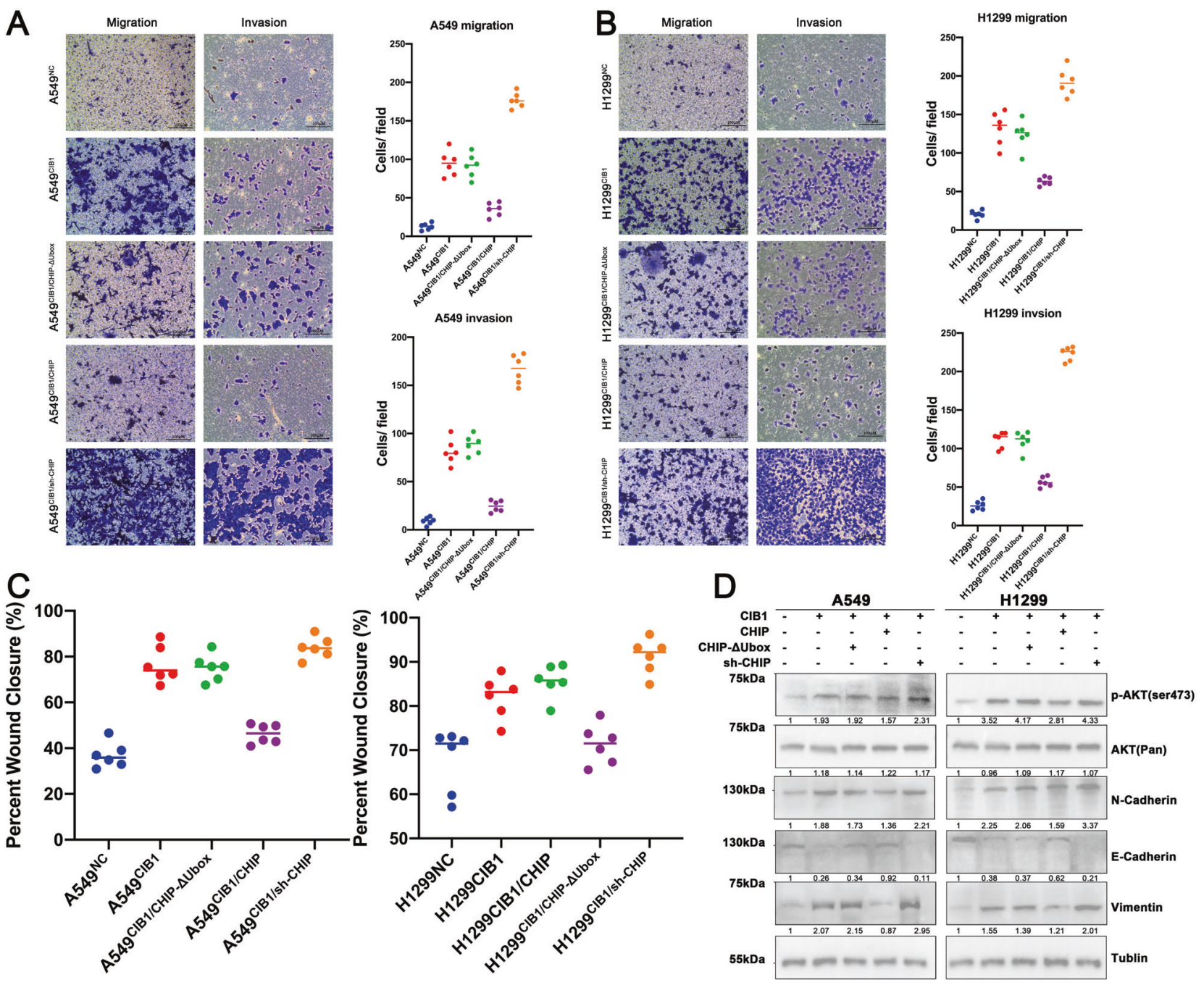

Fig. 6 CHIP upregulation alleviates CIB1 induced LAC migratory capacity and metastatic ability in vitro. A, B Representative images and quantification of the Transwell assays using transfected A549/ H1299 cells. C, D Quantification of the wound healing assays using

further sought to evaluate the ability of CIB 1 in regulating the invasion and migration of LAC cells. The wound healing assays and transwell assays showed that LAC cell lines with CIB1 could significantly affect invasive and migration ability. AKT is a serine/threonine-protein kinase that transmits extracellular stimuli and involved in a series of physiological activities such as apoptosis, proliferation, and differentiation [21]. The PI3K/AKT signaling pathway also is closely related to the development, invasion, and metastasis of human tumors [6, 22]. This study found that overexpression of CIB1 in LAC could also significantly promote AKT activation. Studies have found that abnormal activation of NF- $\mathrm{KB}$, PI3K/Akt, Notch, Wnt/ $\beta$-catenin, TGF- $\beta$, integrin, JAK/STAT3, and Hedgehog can lead to EMT [23-26]. Epithelial-mesenchymal transition (EMT) refers to the morphological changes of epithelial cells to stromal cells or fibroblasts. Abnormal activation of EMT transfected A549/H1299 cells. E Western blot assay showing the phosphorylation of AKT and EMT enhanced by CIB1. Restoration of CHIP attenuated this elevation in LAC cells.

plays an essential role in the development of LAC [27-29]. The previous report has found that Integrin, a binding protein of CIB1, plays an important role in the development of lung cancer by participating in EMT [26]. In the advanced non-small cell lung cancer, silencing E-cadherin can promote the transformation of cells into EMT, while the restoration of E-cadherin expression can actively restore the invasion or migration of tumor cells [30]. While $\mathrm{N}$-cadherin and Vimentin act as mesenchymal cell expression molecules and upregulation of their expression promotes metastasis of LAC [31, 32]. We found that a marked change in the expression of Vimentin, $\mathrm{N}$-cadherin, and E-cadherin when CIB1 expression change. It indicates that CIB1 may promote EMT in LAC cells by activating the PI3K/AKT signaling pathway.

To find proteins that interact with CIB1, we used electrospray mass spectrometry finding that CHIP proteins can 
A

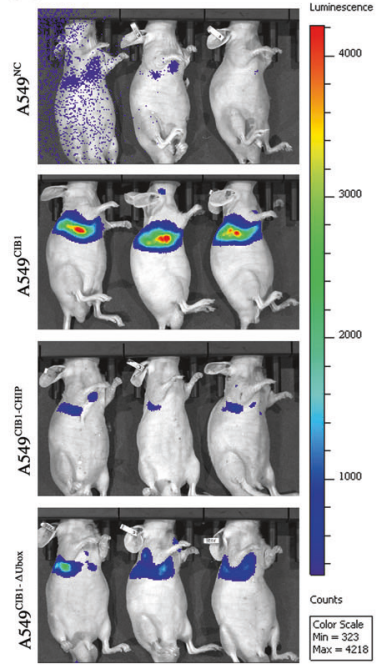

D $A 549^{\mathrm{NC}}$
B

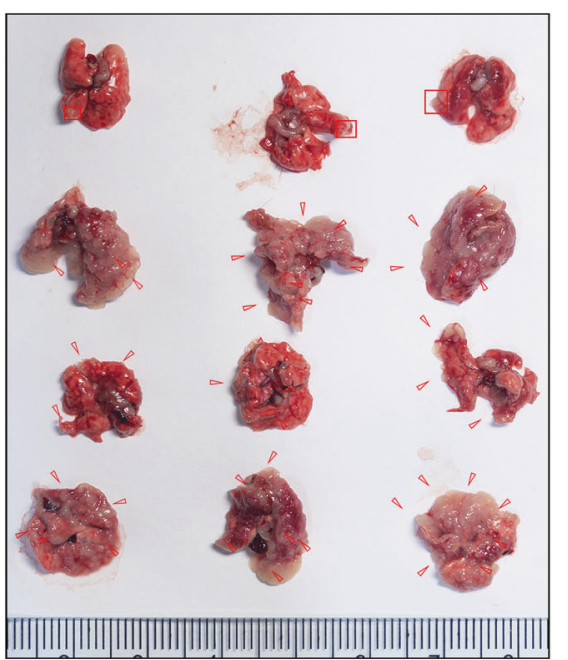

C

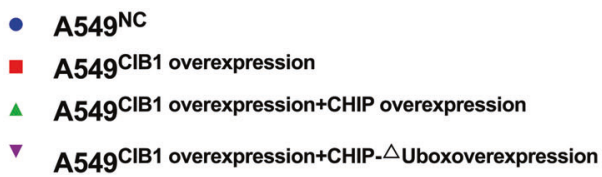

จ A549CIB1 overexpression+CHIP- $\triangle$ Uboxoverexpression

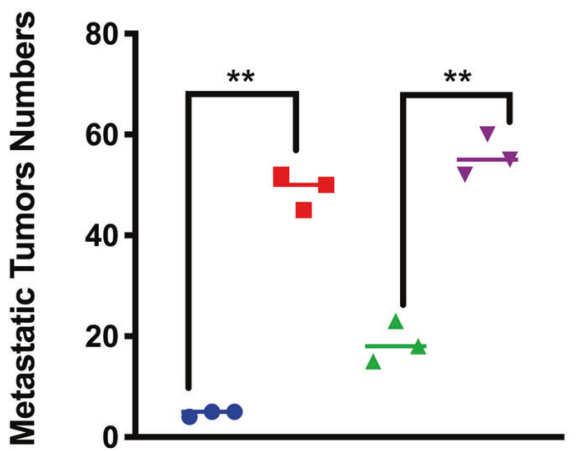

$\mathrm{E}$

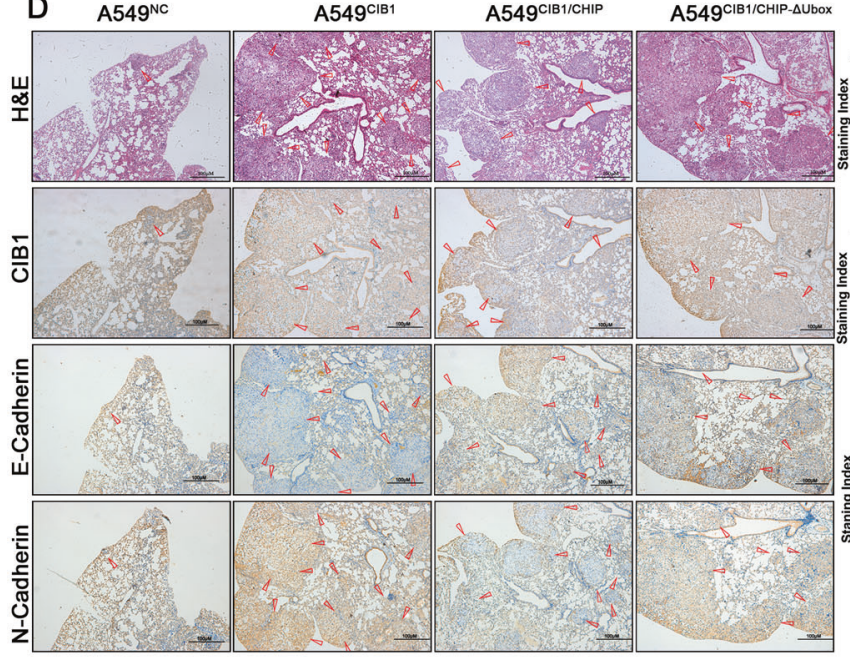

Fig. 7 CHIP upregulation alleviates CIB1 induced LAC migratory capacity and metastatic ability in vivo. A Representative luciferase images and quantification of average luciferase intensity of lungs in the i.v. metastasis assay. B, C Representative photographs and quantification of metastatic tumor nodes in mouse lungs from the i.v. metastasis assay. D Representative immunohistochemically stained

interact with CIB1. CHIP expresses as E3 ubiquitinated ligase, which targets many mature proteins for ubiquitination and proteasomal degradation [33, 34]. CHIP has three functional domains, in which the Ubox domain in the Cterminus provided its E3 ligase activity, which makes CHIP participate in the ubiquitination and degradation process of proteins and plays a broad and important role in the biological process of cells $[11,35,36]$. Many tumor-associated proteins are substrates for CHIP: C-Myc, Ron, ASK1, ErbB2/her2, p53, ASK, etc. [37-43]. Studies have shown that CHIP can negatively regulate the vascular endothelial growth factor pathway, inhibit angiogenic ability, and thus inhibit distant metastasis of tumors $[44,45]$. Also, CHIP can selectively regulate the epidermal growth factor receptor of

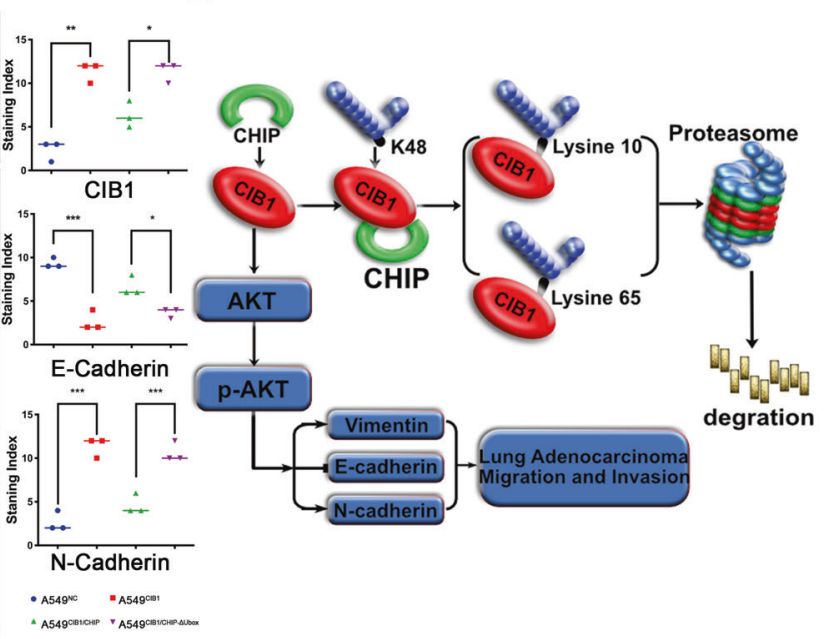

images of lung tissue using anti-CIB1, anti-E-cadherin, and $\mathrm{N}$ cadherin antibody. E CHIP-mediated CIB1 ubiquitination regulated epithelial-mesenchymal and tumor metastasis in lung adenocarcinoma through AKT pathway. ${ }^{*} P<0.05$ vs negative control (NC) group, $* * P<0.01$ vs NC group.

LAC mutants through ubiquitination, thereby affecting the efficacy of chemotherapy [46]. Here we uncover that CIB1 is a new substrate for CHIP. A negative correlation between the expression of CHIP and CIB1 can be found both in cells and tissues. CHIP can affect the stability of CIB1. Notably, by using proteasomal (MG132) showed that CHIPdependent destabilization of CIB1 primarily occurs in the proteasome. Then, the WB followed by co-precipitation show an increasing ubiquitinated CIB1 after CHIP overexpression. However, the CHIP- $\Delta$ Ubox which lose the E3 ligase activity lose the ability to decrease CIB1 expression through facilitated the ubiquitination of CIB1.

Since Lysine (K) residues are ubiquitin acceptor sites for ubiquitin [47], we next sought to find the possible Lysine 
for CIB1 ubiquitination. In this article, we found that K10 and K65 were potential targets for CIB1 ubiquitinate bind site. After K10 and K65 were co-mutation in CIB1, the ubiquitinated CIB1 was decreased.

After finding that CHIP facilitated CIB1 degradation, we then try to test and verify whether CHIP upregulation can alleviate CIB1 induced LAC migratory capacity and metastatic ability. Previous researchers have found that CHIP was expressed in various tumors [33, 44]. In breast cancer, ovarian carcinoma, colorectal cancer, and renal carcinoma, CHIP appears to function as a suppressor of tumorigenesis [48-51]. Here, we found that the regulation of CHIP expression was proved to attenuate the ability of migration and invasion caused by CIB1 in LAC cells both in vivo and vitro. Moreover, The EMT markers, including Vimentin, E-cadherin, N-cadherin, also change with CHIP overexpression. Also, the phosphorylation of AKT was significantly repressed after CHIP transfection. In accordance with our finding, Zhang et al. have demonstrated that CHIP have the function of suppression of lung cancer metastasis [52]. Taken together, our results indicated that CHIP could suppress the EMT process in LAC cells by targeting CIB1 through the AKT pathway.

In summary, our results showed that CIB1 was significantly increased in LAC and correlated with poor prognosis of LAC patients. CIB1 could promote cell invasion and migration both in vitro and in vivo. CHIP could degrade CIB1 by facilitated CIB1 polyubiquitination of CIB1. K10 and K65 were the Lysine $(\mathrm{K})$ residues for CHIP mediated CIB1 degradation. In conclusion, we have carried out research from molecular, cellular, animal models, and clinical specimens to elucidate the molecular mechanism of the interaction between CIB1 and CHIP in inducing migration and invasion of lung adenocarcinoma cells, and to provide experimental evidence for the treatment of new targets for lung adenocarcinoma. Also, we provide a theoretical basis and innovative ideas for the possible construction of a possible treatment strategy for lung adenocarcinoma of CIB1.

Acknowledgements This work was supported by grants from National Natural Science Foundation of China (81372515), National Natural Science Youth Foundation of China (81602027, 81702928), Natural Science Foundation General Program of Hunan Province (2018JJ3821), Natural Science Foundation Youth Program of Hunan Province (806288981033), Project of Scientific Research Plan of Hunan Provincial Health Commission (C2019185) and the Youth Foundation of Xiangya Hospital Central South University (2015Q01).

\section{Compliance with ethical standards}

Conflict of interest The authors declare that they have no conflict of interest.

Publisher's note Springer Nature remains neutral with regard to jurisdictional claims in published maps and institutional affiliations.
Open Access This article is licensed under a Creative Commons Attribution 4.0 International License, which permits use, sharing, adaptation, distribution and reproduction in any medium or format, as long as you give appropriate credit to the original author(s) and the source, provide a link to the Creative Commons license, and indicate if changes were made. The images or other third party material in this article are included in the article's Creative Commons license, unless indicated otherwise in a credit line to the material. If material is not included in the article's Creative Commons license and your intended use is not permitted by statutory regulation or exceeds the permitted use, you will need to obtain permission directly from the copyright holder. To view a copy of this license, visit http://creativecommons. org/licenses/by/4.0/.

\section{References}

1. Chen W, Zheng R, Baade PD, Zhang S, Zeng H, Bray F, et al. Cancer statistics in China, 2015. CA: Cancer J Clin. 2016;66:115-32.

2. Zhou C. Lung cancer molecular epidemiology in China: recent trends. Transl Lung Cancer Res. 2014;3:270.

3. Siegel R, Ma J, Zou Z, Jemal A. Cancer statistics, 2014. CA Cancer J Clin. 2014;64:9-29.

4. Naik UP, Patel PM, Parise LV. Identification of a novel calciumbinding protein that interacts with the integrin?IIb cytoplasmic domain. J Biol Chem. 1997;272:4651-4.

5. Freeman TC Jr, Black JL, Bray HG, Dagliyan O, Wu YI, Tripathy A, et al. Identification of novel integrin binding partners for calcium and integrin binding protein 1 (CIB1): structural and thermodynamic basis of CIB1 promiscuity. Biochemistry. 2013;52:7082-90.

6. Black JL, Harrell JC, Leisner TM, Fellmeth MJ, George SD, Reinhold D, et al. CIB1 depletion impairs cell survival and tumor growth in triple-negative breast cancer. Breast Cancer Res Treat. 2015;152:337-46.

7. Junrong T, Huancheng Z, Feng H, Yi G, Xiaoqin Y, Zhengmao L, et al. Proteomic identification of CIB1 as a potential diagnostic factor in hepatocellular carcinoma. J Biosci. 2011;36:659-68.

8. Zhang P-F, Zeng G-Q, Hu R, Li C, Yi H, Li M-Y, et al. Identification of flotillin-1 as a novel biomarker for lymph node metastasis and prognosis of lung adenocarcinoma by quantitative plasma membrane proteome analysis. J Proteom. 2012;77:202-14.

9. Zheng N, Shabek N. Ubiquitin ligases: structure, function, and regulation. Annu Rev Biochem. 2017;86:129-57.

10. Ebner P, Versteeg GA, Ikeda F. Ubiquitin enzymes in the regulation of immune responses. Crit Rev Biochem Mol Biol. 2017;52:425-60.

11. Sha Y, Rao L, Settembre C, Ballabio A, Eissa NT. STUB1 regulates TFEB-induced autophagy-lysosome pathway. Embo J. 2017;36:e201796699.

12. Huang WG, Cheng AZ. Targeted proteomic analysis of 14-33sigma in nasopharyngeal carcinoma. Int $\mathrm{J}$ Biochem Cell Biol. 2010;42:137-47.

13. Ai-Lan C, Wei-Guo H, Zhu-Chu C, Fang P, Peng-Fei Z, Mao-Yu $\mathrm{L}$, et al. Identification of novel nasopharyngeal carcinoma biomarkers by laser capture microdissection and proteomic analysis. Clin Cancer Res. 2007;14:435-45.

14. Cheng GZ, Park S, Shu S, He L, Kong W, Zhang W, et al. Advances of AKT pathway in human oncogenesis and as a target for anticancer drug discovery. Curr Cancer Drug Targets. 2008;8:2-6.

15. Larue L, Bellacosa A. Epithelial-mesenchymal transition in development and cancer: role of phosphatidylinositol $3^{\prime}$ kinase/ AKT pathways. Oncogene. 2005;24:7443-54.

16. Leisner TM, Moran C, Holly SP, Parise LV. CIB1 prevents nuclear GAPDH accumulation and non-apoptotic tumor cell death via AKT and ERK signaling. Oncogene. 2013;32:4017. 
17. Yoon KW, Cho J-H, Lee JK, Kang Y-H, Chae JS, Kim YM, et al. CIB1 functions as a $\mathrm{Ca}^{2+}$-sensitive modulator of stress-induced signaling by targeting ASK1. Proc Natl Acad Sci. 2009;106: 17389-94.

18. Yuan W, Leisner TM, McFadden AW, Wang Z, Larson MK, Clark S, et al. CIB1 is an endogenous inhibitor of agonist-induced integrin $\alpha I I b \beta 3$ activation. J Cell Biol. 2006;172:169-75.

19. Jarman KE, Moretti PA, Zebol JR, Pitson SM. Translocation of sphingosine kinase 1 to the plasma membrane is mediated by calcium-and integrin-binding protein $1 . \mathrm{J}$ Biol Chem. 2010;285:483-92.

20. Son S, Byun J, Roh S, Kim S, Mook-Jung I. Reduced IRE1 $\alpha$ mediates apoptotic cell death by disrupting calcium homeostasis via the InsP3 receptor. Cell Death Dis. 2014;5:e1188.

21. Chang R-M, Xu J-F, Fang F, Yang H, Yang L-Y. MicroRNA-130b promotes proliferation and EMT-induced metastasis via PTEN/pAKT/HIF-1 $\alpha$ signaling. Tumor Biol. 2016;37:10609-19.

22. Alexandre M, Lin ZP, Ratner ES. Abstract 850: the Pi3K/Akt pathway mediates epithelial-mesenchymal transition (EMT) and malignant progression in BRCA-defective epithelial ovarian cancer. Cancer Res. 2017;77(13 Supplement):850.

23. Bao B, Azmi AS, Ali S, Ahmad A, Li Y, Banerjee S, et al. The biological kinship of hypoxia with CSC and EMT and their relationship with deregulated expression of miRNAs and tumor aggressiveness. Biochim Biophys Acta-Rev Cancer. 2012;182:272-96.

24. Espinoza I, Miele L. Deadly crosstalk: notch signaling at the intersection of EMT and cancer stem cells. Cancer Lett. 2013;341:41-5.

25. Tirino V, Camerlingo R, Bifulco K, Irollo E, Montella R, Paino F, et al. TGF- $\beta 1$ exposure induces epithelial to mesenchymal transition both in CSCs and non-CSCs of the A549 cell line, leading to an increase of migration ability in the CD133+ A549 cell fraction. Cell Death Dis. 2013;4:e620.

26. Eberlein C, Rooney C, Ross S, Farren M, Weir H, Barry S. ECadherin and EpCAM expression by NSCLC tumour cells associate with normal fibroblast activation through a pathway initiated by integrin $\alpha v \beta 6$ and maintained through TGF $\beta$ signalling. Oncogene. 2015;34:704.

27. Pradella D, Naro C, Sette C, Ghigna C. EMT and stemness: flexible processes tuned by alternative splicing in development and cancer progression. Mol Cancer. 2017;16:8.

28. Lamouille S, Xu J, Derynck R. Molecular mechanisms of epithelial-mesenchymal transition. Nat Rev Mol Cell Biol. 2014;15:178.

29. Tan X, Banerjee P, Guo H-F, Ireland S, Pankova D, Ahn Y-h, et al. Epithelial-to-mesenchymal transition drives a pro-metastatic Golgi compaction process through scaffolding protein PAQR11. J Clin Investig. 2017;127:117-31.

30. Mateen S, Raina K, Agarwal C, Chan D, Agarwal R. Silibinin synergizes with histone deacetylase and DNA methyltransferase inhibitors in upregulating E-cadherin expression together with inhibition of migration and invasion of human non-small cell lung cancer cells. J Pharmacol Exp Ther. 2013;345:206-14.

31. Zhang X, Liu G, Kang Y, Dong Z, Qian Q, Ma X. N-cadherin expression is associated with acquisition of EMT phenotype and with enhanced invasion in erlotinib-resistant lung cancer cell lines. PloS ONE. 2013;8:e57692.

32. Li X, Zhu J, Liu Y, Duan C, Chang R, Zhang C. MicroRNA-331$3 p$ inhibits epithelial-mesenchymal transition by targeting ErbB2 and VAV2 through the Rac1/PAK1/ $\beta$-catenin axis in non-smallcell lung cancer. Cancer Sci. 2019;110:1883.

33. Paul I, Ghosh MK. A CHIPotle in physiology and disease. Int $\mathbf{J}$ Biochem Cell Biol. 2015;58:37-52.
34. Edkins AL. CHIP: a co-chaperone for degradation by the proteasome. Subcell Biochem. 2015;78:219-42.

35. Ballinger CA, Connell P, Wu Y, Hu Z, Thompson LJ, Yin L-Y, et al. Identification of CHIP, a novel tetratricopeptide repeatcontaining protein that interacts with heat shock proteins and negatively regulates chaperone functions. Mol Cell Biol. 1999; 19:4535-45.

36. Sun C, Li H-L, Shi M-L, Liu Q-H, Bai J, Zheng J-N. Diverse roles of C-terminal Hsp70-interacting protein (CHIP) in tumorigenesis. J Cancer Res Clin Oncol. 2014;140:189-97.

37. Parsons JL, Tait PS, Finch D, Dianova II, Allinson SL, Dianov GL. CHIP-mediated degradation and DNA damage-dependent stabilization regulate base excision repair proteins. Mol Cell. 2008;29:477-87.

38. Hatakeyama S, Watanabe M, Fujii Y, Nakayama KI. Targeted destruction of c-Myc by an engineered ubiquitin ligase suppresses cell transformation and tumor formation. Cancer Res. 2005;65:7874-9.

39. Germano S, Barberis D, Santoro MM, Penengo L, Citri A, Yarden Y, et al. Geldanamycins trigger a novel Ron degradative pathway, hampering oncogenic signaling. J Biol Chem. 2006;281:21710-9.

40. Hwang JR, Zhang C, Patterson C. C-terminus of heat shock protein 70-interacting protein facilitates degradation of apoptosis signalregulating kinase 1 and inhibits apoptosis signal-regulating kinase 1dependent apoptosis. Cell Stress Chaperones. 2005;10:147-56.

41. Tripathi V, Ali A, Bhat R, Pati U. CHIP chaperones wild type $\mathrm{p} 53$ tumor suppressor protein. J Biol Chem. 2007;282:28441-54.

42. Esser C, Scheffner M, Hohfeld J. The chaperone-associated ubiquitin ligase CHIP is able to target p53 for proteasomal degradation. J Biol Chem. 2005;280:27443-8.

43. Zhou P, Fernandes N, Dodge IL, Reddi AL, Rao N, Safran H, et al. ErbB2 degradation mediated by the co-chaperone protein CHIP. J Biol Chem. 2003;278:13829-37.

44. Kajiro M, Hirota R, Nakajima Y, Kawanowa K, So-ma K, Ito I, et al. The ubiquitin ligase CHIP acts as an upstream regulator of oncogenic pathways. Nat Cell Biol. 2009;11:312-9.

45. Tingting Q, Jiao W, Qingfeng W, Yancheng L, Shijun Y, Zhaoqi $\mathrm{W}$, et al. CHIP involves in non-small cell lung cancer prognosis through VEGF pathway. Biomed Pharmacother. 2016;83:271-6.

46. Chung C, Yoo G, Kim T, Lee D, Lee C-S, Cha HR, et al. The E3 ubiquitin ligase CHIP selectively regulates mutant epidermal growth factor receptor by ubiquitination and degradation. Biochem Biophys Res Commun. 2016;479:152-8.

47. Su D, Jing Z, Wei J, Bowser RK, Khoo A, Liu Z, et al. F-box protein complex FBXL19 regulates TGF 1 -induced E-cadherin downregulation by mediating Rac3 ubiquitination and degradation. Mol Cancer. 2014;13:76.

48. Luan H, Mohapatra B, Bielecki TA, Mushtaq I, Mirza S, Jennings TA, et al. Loss of the nuclear pool of ubiquitin ligase CHIP/ STUB1 in breast cancer unleashes the MZF1-cathepsin prooncogenic program. Cancer Res. 2018;78:canres.2140.016.

49. Wang Y, Ren F, Wang Y, Feng Y, Wang D, Jia B, et al. CHIP/Stub1 functions as a tumor suppressor and represses NF-?B-mediated signaling in colorectal cancer. Carcinogenesis. 2014;35:983-91.

50. Shang Y, He J, Wang Y, Feng Q, Zhang Y, Guo J, et al. CHIP/ Stub1 regulates the Warburg effect by promoting degradation of PKM2 in ovarian carcinoma. Oncogene. 2017;36:4191-200.

51. Min B, Park H, Lee S, Li Y, Choi J-M, Lee JY, et al. CHIP-mediated degradation of transglutaminase 2 negatively regulates tumor growth and angiogenesis in renal cancer. Oncogene 2015;35:3718.

52. Zhang P, Li C, Li H, Yuan L, Dai H, Peng Z, et al. Ubiquitin ligase CHIP regulates OTUD3 stability and suppresses tumour metastasis in lung cancer. Cell Death Differ. 2020. https://doi.org/ 10.1038/s41418-020-0571-7. [Epub ahead of print]. 